\title{
Estimating Fish Densities from Single Fish Echo Traces
}

\author{
Magnar Aksland*
}

\author{
University of Bergen, Department of Biology, P.O. Box 7800, N-5020 Bergen, Norway
}

\begin{abstract}
Estimating mean fish density per unit area has been done by counting single fish echo traces with a split beam echo sounder system in the open sea. A data program has been written that scans the acoustic signals for echo peaks and classify these into echo traces. The program counts the number of echo traces from fish that cross the beam within given distances from the acoustic axis over a known sailing distance, and gives an absolute estimate of fish density. This is obtained by using the off acoustic axis angles of fish that generate resolved echoes to compute the athwartship distance from the vertical acoustic axis to the fish. The program is described and demonstrated on selected files of raw data recorded by the SIMRAD EK 60 split beam system. Estimated fish densities for these files are also obtained by the echo integration method, and the echo trace method gives estimates that agree well with these estimates under sufficiently "good conditions".
\end{abstract}

\section{INTRODUCTION}

Underwater acoustics has a wide area of applications, such as underwater communication, mapping the bottom topography, Doppler speed measurements and so on. Use of sonar and echo sounder to detect fish and map the distribution of fish populations started before the world war 2, [1]. Acoustic abundance estimation of fish followed and started by the echo counting method [2-4], but this was replaced early on by echo integration, a method that has developed further since then and became the standard method of acoustic abundance estimation. The most important reason for this is that the echo integration method has few restrictions on the within-beam fish density. The physical reason that many fish species are easily detected acoustically is because they have swim bladder that account for around 90 percent of their back scattering strength [5]. A theoretical foundation for the echo integration method is given in [6], and a modern software system based on echo integration is described in [7].

The present paper presents a method to estimate fish density by counting single fish echo traces, which is the collection of echoes received from the same target, such as a fish, when it crosses the sound beam of a split beam echo sounder.

There are few references about the use of echo trace methods, or echo tracking, for the purpose of abundance estimation in the literature. In [8], echo traces from salmon and herring was counted in rivers by means of stationary sidescan sonar based on a method given in [9]. This method was further developed by [10]. The actual computer algorithm used is given in [11]. However, detecting echo traces from a moving acoustic platform with a down looking echo sounder in the open sea is not quite the same as using a stationary side scan sonar in rivers.

*Address correspondence to this author at the University of Bergen, Department of Biology, PO Box 7800, N-5020 Bergen, Norway;

E-mail: magnar.aksland@bio.uib.no
Otherwise, echo traces have been detected for purposes other than abundance estimation. Several studies involving echo traces with the split beam systems have been done in Japan, e.g. [12-14] and others.

In Norway a method using stationary split beam systems to study fish behavior has been developed and applied. The method is called target tracking and involves the detection of single fish positions during each ping when the fish are within the beam. Both this method and the echo trace method presented in this paper require the classification of echoes in the echo signal into groups of echoes that come from the same target. For description and use of the target tracking method see $[15,16]$ and references therein.

Estimating fish abundance or density by counting echo traces by a down looking transducer requires a moving acoustic platform where the acoustic beam moves over fish in a straight line. As long as the number of pings transmitted during the fish crossing time is high enough, and echoes from several targets do not overlap seriously, the estimation method based on counting traces may be used in theory.

Although the principles behind the echo trace method using a split beam system are simple, a good program that classifies the echoes in the echo signals into single fish echo traces is a challenge to write. This is because echo parameters observed within a trace often deviate more or less from that expected. The computer program presented here is written from the basis without using the techniques given in [11]. Reasons for this are given in the Discussion.

The method requires sufficiently low within beam fish density so that a "sufficient fraction" of the received echoes are "at least partly" resolved. In addition, the average number of echoes in the traces cannot be too small because then many traces from the beam crossing fish may not be observed. If there is a fraction of fish from which only one echo is received, these are not counted. Suitable values for the sailing speed and ping repetition rate are also required. Although a slow sailing speed will generate traces with many echoes, the sailing speed must be significantly bigger 
than the swimming speeds of the detected fish to avoid the formation of many traces that are fractions of long traces. This happens if the echo parameters from a target change too much relative to that expected from a stationary target.

The purpose of this study was to try the echo trace estimation method in practice and find out more about conditions for successful use.

\section{MATERIAL AND METHODS}

Egil Ona at the Institute of Marine Research (IMR), Bergen, Norway, provided the files of acoustic raw data used to develop this method. These files were recorded on cod and contain a large fraction of resolved echoes.

Recordings from a complete acoustic survey in Lofoten, Northern Norway, were available. The survey was performed in March/April 2004 with the new R/V G. O. Sars. During the survey modern scientific echo sounders operated simultaneously at the five frequencies 18, 38, 70, 120 and 200 $\mathrm{kHz}$, all with split beam transducers mounted close together. All beams were near circular symmetric. The half power beam width was 10.7 degrees for $18 \mathrm{kHz}$ and close to $7 \mathrm{de}$ grees for the other frequencies. All echo sounders used the same pulse length of $1 \mathrm{~ms}$, and the rectified and smoothed echo signal was sampled each $0.256 \mathrm{~ms}$ giving roughly 4-6 samples over one echo pulse. The working principles for the split beam echo sounder system are given by [17].

\section{Principles for a Trace Program}

The program described below is written in standard $\mathrm{C}$, and works with $40 \log$ TVG (Time Varied Gain) digital echo signals. A flow chart of the program is given in Appen$\operatorname{dix}$ A.

Detection of echo peaks in the trace program is simple. If there is a value in the digitized echo signal where both neighbor values are smaller, the biggest value is taken to be the peak value of a possible echo. This is then subject to tests and calculations for being a candidate echo in a trace. The reason for using a simple test to detect possible echo peaks is to avoid losing too many echoes in the first place.

To avoid echoes with very imprecise parameters, echoes to be used must come from a target that is not too far out in the beam, and the beam-compensated peak value should exceed a threshold that must be set by the user. No test is done on echo shape. This means that partly overlapping echoes as well as echoes that partly overlap with the bottom echo are used as possible trace candidates if their peaks appear as local maximums in the echo signal.

The echo parameters Ping number, Echo depth, Alongship angle and Athwartship angle are used to test whether an accepted echo belongs to some unfinished echo trace. In addition to these parameters, the program also computes measures of the stability of the alongship and athwartship angles. These parameters depends on the variation of the values of the phase angles within one echo, and express something about how accurately the off axis angles of an echo are measured by the split beam system. They are used during the computation of weighted average values. The program uses several average values over the echoes of a trace, and these are computed by using higher weights for echoes with pre- cise parameters than the weights of echoes with imprecise parameters.

To decide which of the echoes in a new ping that belong to which of the unfinished traces, the echo parameters of each new echo are compared with the expected echo parameters of the next echo in each trace. These expected parameter values are computed from the parameters of the echoes already in the trace, the sailing speed of the ship, as well as the transmit time of the ping where the candidate echo is found. For each new accepted candidate echo, a "distance" from the expected parameters of the next echo is computed for every unfinished trace. This distance is simply a linear function of the absolute deviations between the expected echo parameters and the observed parameters of a new echo.

A new echo is taken to belong to it's closest trace if the distance to this trace is less than a given value, and if the deviations of each single parameter are also less than particular given values. This trace is updated with the new echo if the distance test is passed. If the distance test for a given new echo fails, or if there are no traces containing echoes, the echo is accepted as the first echo of a new trace provided that the corresponding target is not too far behind in the beam.

Setting of proper values for the maximum distance to a trace as well as the maximum values for each parameter deviation are important for the program to work satisfactorily. It was necessary to run the program with different values of these variables to find favorable values.

It may happen that more than one echo in the next ping has the same trace as the closest. In that case the echo with the closest distance is taken as the next echo in the trace. The next closest echo is then checked against the other unfinished traces, and if it is the closest echo to another trace, it is taken as the next echo there instead of the echo that was computed as the closest in the first instance.

All unfinished traces are tested for being finished before each new ping, that is, if the difference between the present ping number and the ping number of the last echo in the trace exceeds a given value. As soon as a trace is finished, the weighted average depth and athwartship angle of the target generating the trace are computed. These values are then used to compute the athwartship distance between the target and the vertical acoustic axis. The purpose is to be able to count the number of traces, or targets, within different athwartship distances to the acoustic axis. The weighted average echo amplitude over the trace echoes is also computed.

Selected echo parameters of the echoes in an echo trace are shown graphically in Fig. (1). As this method will work with any chosen level of the digitized echo signal, the axis for Echo amplitude has no numeric labels.

The sailing speed was $6.8 \mathrm{~m} / \mathrm{s}$, and the variations in the echo parameter values are likely to be caused by movements of the target.

The echo parameters of each echo in two selected traces are shown in Appendix B.

\section{Expected Parameter Values for the Next Echo in a Trace}

The depths of the echoes in a trace should ideally be constant, but in practice there is always some variation. The ab- 

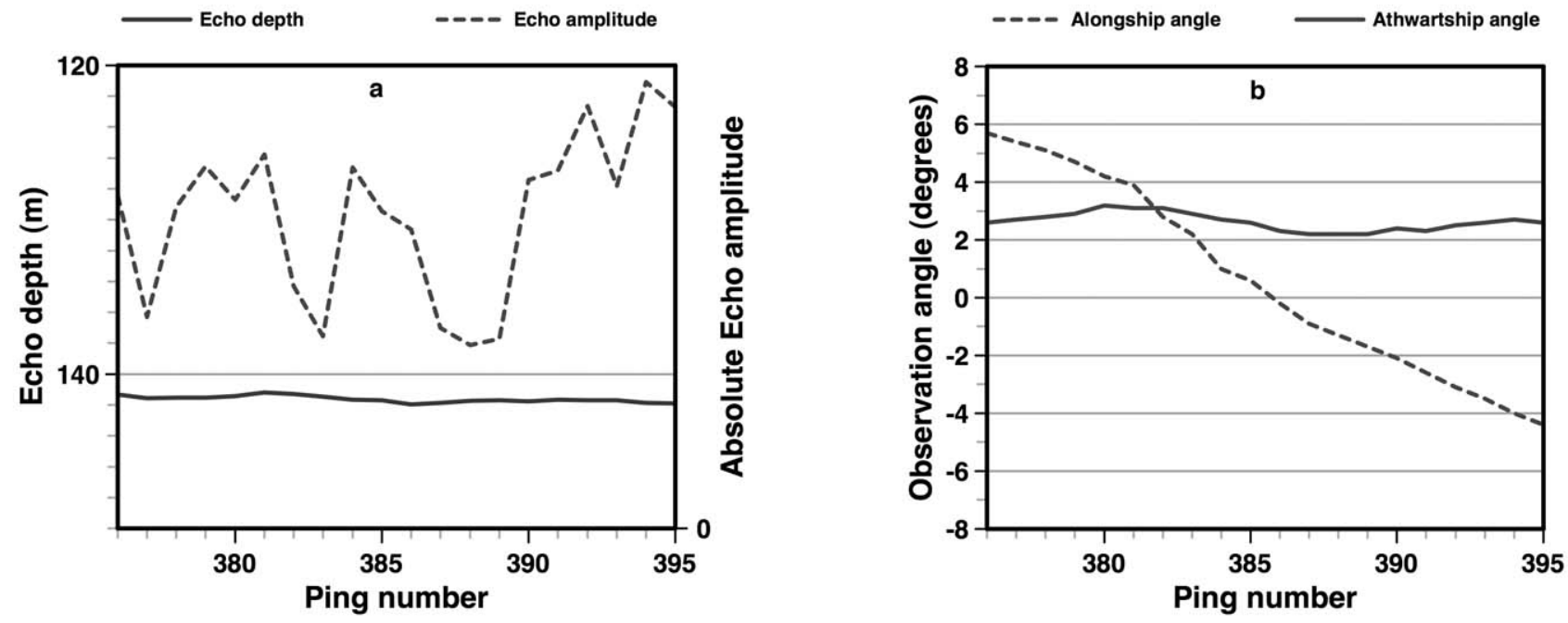

Fig. (1). Echo depth and absolute beam compensated echo amplitude (a), alongship and athwartship angles (b), as functions of the ping numbers of a long echo trace containing 20 echoes.

solute echo peak amplitudes show big variations in accordance with the long known scattering properties of fish [18], as well as biases caused by overlapping echoes and errors in the beam compensations.

If the acoustic platform is moving along a horizontal straight line, as is normal in acoustic surveys, the alongship angle of a fish will start with a positive value and decrease for each ping, ending the trace with negative values (behind the beam).

The athwartship angles are, on the contrary, independent of the forward movement and should ideally be constant for the echoes in a trace from a stationary target.

However, all parameters show variations that deviate from their ideal values. Movement of the fish that generates the trace may cause variations in echo depth and angles. Uncompensated heave, pitch and roll movements of the acoustic platform are another cause. The program described here compensates for unwanted platform movements when values for the platform's heave, pitch and roll are contained in the acoustic data. After beam compensation of the echo intensity the echo parameters are transformed to a fixed spherical reference system without variations in heave, pitch and roll by subtracting these values from the corresponding detected depths, alongship and athwartship angles of the echoes.

The detection of the off axis angles of an echo may be subject to errors, in particular when these angles are big, and when echoes overlap. Errors in the off axis angles will also lead to errors in the beam compensation of the echo peak values, thus producing biased target strength values.

In the light of these properties the expected values of the parameters of the next echo in a trace are based on particular average values of the parameters of echoes already in the trace. The weight function

$$
w=\left(1+\theta_{r}^{2}+\Delta a_{l}+\Delta a_{t}\right)^{-1}
$$

is used in different ways for each echo parameter, where $\theta_{r}$ is the beam width relative angle between the acoustic axis and the direction between the transducer and the scattering fish, and $\Delta a_{l}$ and $\Delta a_{t}$ are the instability indices of the alongship and athwartship angles of the echo.

The angle instability indexes used here are simply the average absolute deviation from the mean of the three most even successive angle values in the echo signal containing the site of the echo peak. There are exactly three different sets of three successive angle values that contain the site of the peak. In general, the number of successive angles used should be a constant fraction of the number of signal sample values over one pulse length.

The angle $\theta_{r}$ is defined relative to the half power opening angle of the transducer beam, and should be specified so that $\theta_{r}$ only contributes significantly in (1) at angles in the outer region of the beam where errors in observation angles are significant. So far, a constant of proportion making $\theta_{r}$ equal to one when the associated absolute angle $\theta$ is equal to $\sqrt{2}$ times the half power beam width, has been used. The small angle approximation

$$
\theta \approx \sqrt{a_{l}^{2}+a_{t}^{2}}
$$

is used in computations. This works well with the narrow beams normally used in acoustic surveys.

The expected depth of the next echo is computed as a weighted average depth of the echoes already in the trace. Although the weight function (1) may not be the most appropriate for the echo depths, these depths are computed by $z=r \cos \theta$, where $r$ is range and $z$ is depth. The depth therefore depends on the detection angle but will be rather insensitive to errors in this angle.

But as some drift in the echo depths is often observed within traces, the weights are also made dependent on the echo number within the trace. The echo number of an echo is equal to one more than the difference between the ping numbers of that echo and the first echo in the trace. For each new trace echo, the weight (1) is multiplied by a factor raised to 
the power of the echo number, thus reducing the relative weights of the first echoes considerably. Factor values around 1.5 have been used.

The expected beam compensated echo amplitude of the next echo is computed as a weighted average over the echoes in the trace using (1), without multiplication with factors that reduce the weights of the first echoes.

A simple model for the expected alongship angle of the next echo in a trace may be given by the alongship angle of the last echo plus the expected change $\Delta a_{l}$ in the alongship angle over the time period between the last and the next echo in the trace. The following relation is obtained from geometrical considerations.

$$
\Delta a_{l} \approx \sin \left(\Delta a_{l}\right)=v_{s} \Delta t / z
$$

where $v_{s}$ is the sailing speed, $\Delta t$ is the time between the next and the last echo and $z$ is the depth of the corresponding target. The approximation is good for small values of $\Delta a_{l}$, and is used in the program. This model assumes that the target has a constant position during the observation time of the trace. Since fish are able to move, and also because angles observed by the split beam system may be subject to errors, a modified version of the above is used on traces with more than one echo. This is based on a weighted linear regression of the alongship angle for the existing echoes against the transmit time associated with each echo.

The weight function (1) multiplied with factors giving low weight to the first echoes, as for the echo depth, is used in the regression. This regression is first used to predict the average, or expected, alongship angle at the time of the last echo already in the trace. An alongship angle slope with respect to time between the times of the last and the next echo is then computed. This slope is a special weighted average of the slope of the regression and the corresponding slope $v_{s} / z$ given by (2) above. The slope is given by

$$
s=\frac{m s_{T}+(n-1) s_{R}}{m+n-1},
$$

where $m$ is a user specified parameter, $n$ is the present number of echoes in the trace, $s_{T}$ is the theoretical slope $v_{s} / z$ and $s_{R}$ is the slope from the regression. For traces with only one echo, the slope (3) is equal to the theoretical slope (no regression slope exists), while the regression slope has an increasing influence on (3) when the number of echoes in the trace increases. The idea behind this is that the first echoes in a trace may give the wrong slope. Fig. (2) shows the variations in $S_{T}$ and $S_{R}$ as a function of the present number of echoes in the traces from a selected run.

Fig. (2) shows that the regression slope is very variable for traces containing few echoes. This variation is likely to be caused by errors in the alongship angle. Most traces start with echoes from targets far out in the beam where errors in the detection angles are normal. The presence of partly overlapping echoes may also cause significant errors in the detec-

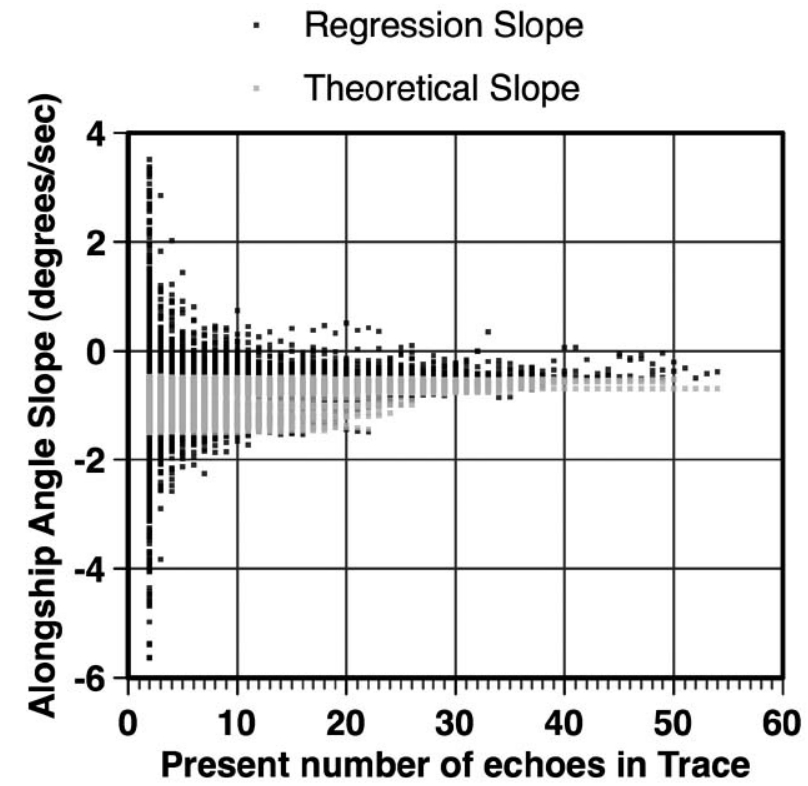

Fig. (2). Variations in the theoretical (grey) and regression (black) slopes for the alongship angle per unit time over targets at different present number of echoes in the traces.

tion angles. When there are several echoes in the trace, these errors tend to average down, and differences between $s_{T}$ and $S_{R}$ are more likely caused by movements of the targets.

The expected alongship angle of the next echo of a trace is computed when a candidate for the next echo is observed, from a particular alongship angle value at the time of the last echo, the slope (3) and the time between the last and the next echo. The above mentioned alongship angle has a value between the observed and regressed alongship angle at the time of the last echo.

Finally, the expected athwartship angle of the next echo is computed in a similar way as for the expected echo depth, although another factor for computing higher weights for the last echoes may be used.

\section{Choosing the Next Echo in Unfinished Traces}

There are professional methods to determine which echoes that come from the same target. These methods are also applied in radar technology in addition to the field of underwater acoustics. The methods make use of Kalman filters and are described in [19]. However, although [19] is a comprehensive book, it is not easy to find appropate techniques there. Therefor, the present method does not make use of [19]. It is easier to use the experience with the echo and angle signals in the available files of raw data to write basic code for an independent method that is likely to be faster and easier to develop further than the professional method. The present method may be described as follows:

As soon as a new candidate echo is accepted, a deviation value between each echo parameter and the corresponding expected parameter for the next echo in each unfinished trace are computed, as well as a distance value given as a linear combination of the parameter deviations. 
The individual parameter deviations are given as the absolute value of the difference between the expected and observed values except for the echo amplitude where the absolute value of the natural logarithm of the ratio between the observed and expected echo amplitude is used. The factors of multiplication with which the different deviations are included in the distance function may be taken as parameters of the program, but they should reflect the way each deviation varies for echoes belonging to a trace.

Based on some preliminary statistics on observed traces, the factors of multiplication for the different parameters in the distance function should be almost equal to each other. They are therefore set equal to one in the program. However, improvements are possible here. Under poorer signal to noise ratios the phase angles will be more variable caused by bigger observation errors. This suggests that the factors of multiplication for the deviations of the alongship and athwartship angles might better be dependent on signal to noise ratio, or on the range.

\section{Counting Finished Echo Traces}

Each finished trace is counted if it contains at least $n_{\min } \geq 2$ echoes, where the value of $n_{\min }$ can be set, and if it passes a test on the echo amplitudes within the trace. The natural value for $n_{\min }$ would be 2 . However, experience with the program so far indicates that the number of short traces is often overestimated. In particular, the number of two-echo traces seems in general to be too big, but three-echo traces and even longer traces may also be somehow overestimated in number. Therefore, values of 3 or 4 for $n_{\min }$ have most often been used. Cases where use of $n_{\min }=4$ has been successful is when the average trace length is big. It seems that the loss of the true number of traces with fewer echoes than $n_{\text {min }}$ is roughly compensated by the overestimated number of traces with more echoes. Arguments for this are given in the Results and Discussion sections.

The distribution of trace lengths may be derived theoretically based on the beam width, range, sailing speed, ping repetition rate, and selected assumptions. One work is given by [20], but the observed trace lengths during the use of the echo trace method cannot be expected to follow the theoretical distributions since movements of fish, as well as observations of traces over different regions of beam angles, occur.

Traces are made for all species represented in the acoustic recording, and usually a target species will be more or less mixed with other species. It turned out to be difficult to filter out traces from unwanted species if their distribution of echo amplitudes is considerably overlapping with the echo amplitudes of the target species. As this method was applied on cod, the majority of all unwanted traces came from species with lower echo amplitudes than cod. It was thus decided to accept that a trace came from cod if the mean echo amplitude within the trace exceeded a particular threshold.

To find a value for this threshold, a histogram was plotted of the Target Strength $(\mathrm{dB})$ of the mean amplitudes within all traces that were found. A histogram from one of the files of raw data is shown in Fig. (3).

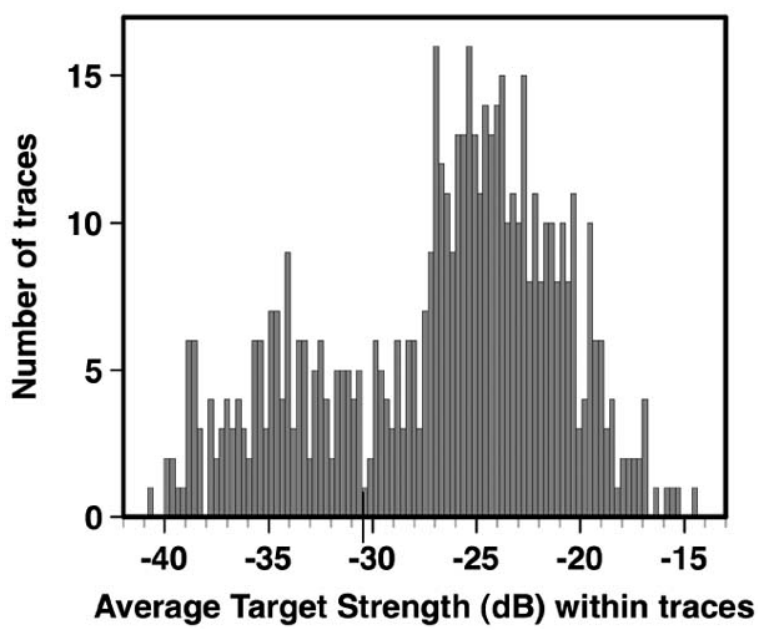

Fig. (3). The distribution of the Target Strengths (dB) corresponding to the mean echo amplitudes within a trace over all traces.

This distribution is the combination of two distributions, one for cod and the other for all other species. A likely place to split these distributions is at the value $-30.5 \mathrm{~dB}$ in Fig. (3). Experience has demonstrated that if there are low values in this distribution near $15 \mathrm{~dB}$ below the maximum, the trace estimate will have a value close to the integrator estimate if the threshold is set here.

The Target Strengths in Fig. (3) are biased because of overlapping echoes. The biggest source of bias is most likely wrong beam compensations caused by biased phase angles. These biases give the observed distributions as that in Fig. (3) wider range than the corresponding true distribution, but they also make it more difficult to determine a good value for the threshold. It is, however, only possible to use the trace counting method with success when a likely value of a threshold to filter away traces from unwanted species can be seen from histograms corresponding to Fig. (3).

Other ways to filter out traces from unwanted species have been tried. A threshold for the maximum echo amplitude within the traces was more difficult to set because maximum amplitudes varied too much between traces.

By running the program that counts the traces with mean amplitudes above $-30.5 \mathrm{~dB}$, as indicated in Fig. (3), an estimate of mean fish density is obtained that is consistent with the corresponding estimates based on echo integration. The program also estimates fish density by echo integration using integrated single echo intensities to convert integrator values to fish density. A version of the program described in [21] was added as an integrator routine to the trace program.

\section{Preparations for Running the Trace Program}

The results of running the trace program on selected files of raw data are presented in the next section. As no part of the survey that recorded the files of raw data was prepared for the use of the echo trace method, it was necessary to go through all available files and pick out those few that had the type of recording, sailing speed, ping repetition rate, and weather conditions that were suitable for using the trace method to estimate fish density. Weather conditions were checked by plotting the time variations in the heave, pitch and roll data. For some files the roll angle had variations of 
the order of, or bigger than, the beam width. In such circumstances, many trace echoes would be lost for some periods of time because the target fish are outside the range of angles where candidate echoes are accepted.

Many good-looking recordings from echograms were discarded because a value for a threshold to filter away traces from small individuals was not apparent from the average Trace Target Strength histogram. To increase the possibility to see a likely value for this threshold, it is important to exclude as much as possible of the region outside the target recording for search of trace echoes. In the end, only 9 files were selected for being appropriate for the trace method, but only a fraction of these files are used in this paper.

A flow chart of the trace program together with further technical information are given in Appendix A. A table showing the parameter values of the echoes in two selected traces is given in Appendix $\mathbf{B}$.

\section{RESULTS}

The files of raw data used here represent different fish densities and compositions, fish depths, sailing speeds and ping repetition rates. To illustrate the estimation procedure, some of the results are presented by figures showing echograms at $38 \mathrm{kHz}$, selected threshold values and distribution of integrated single echoes. All files were run at $38 \mathrm{kHz}$ and
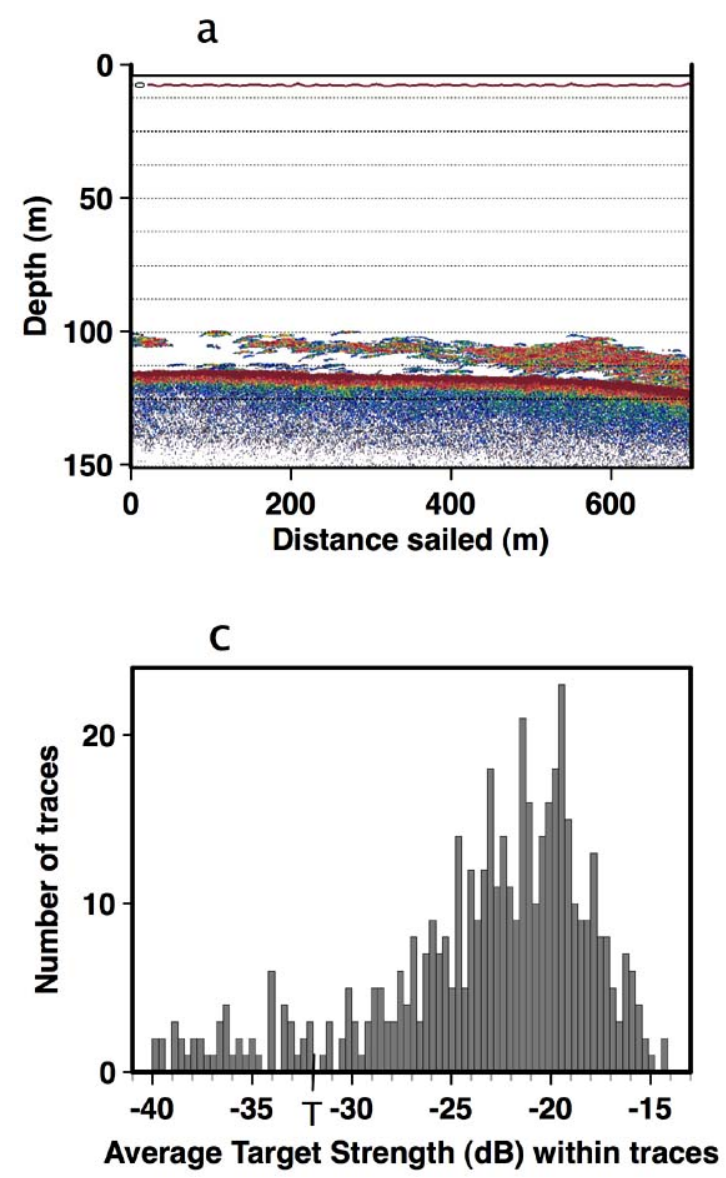

some also at other frequencies. Each file had to be run several times while adjusting different parameter values. This is not a big disadvantage as the execution time is of the order of a few seconds. With the modern acoustic system used in this investigation, the noise levels were quite low and did not cause any problem. Details about the noise are shown in Appendix A.

The first file represents a dense recording with some overlapping echoes over a bottom depth of $110-120 \mathrm{~m}$. The sailing speed was between 1.8 and $2.2 \mathrm{~m} / \mathrm{s}$ and the ping repetition rate around 2.5 pings per second. The distance sailed was $779 \mathrm{~m}$. Part of the echogram and integrated single echoes is shown in Fig. (4) together with histogram of the average target strength within the traces and the cumulative distribution of the average athwartship distances between the acoustic axis and the traces.

The single echo diagram was used to choose a threshold value to filter out weak echoes from species of smaller organisms when estimating the conversion factor between the fish density estimate and the total echo integrator value. This recording comes from cod that were mixed with very few smaller organisms that are easily filtered away by proper threshold values. For more details on this, reference is made to [21]. The integrator estimate is 0.057 fish per square meter.
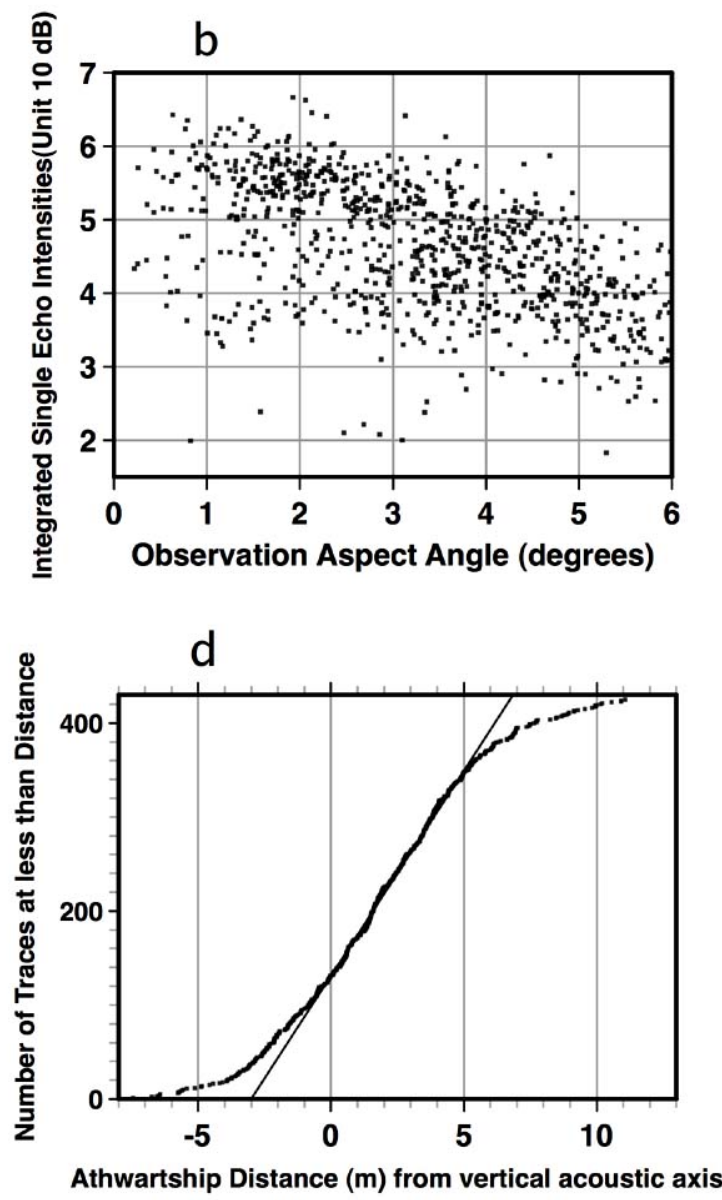

Fig. (4). Echogram with $12.5 \mathrm{~m}$ between the depth lines (a), integrated single echo intensities (b), distribution of average trace Target Strengths (c) and cumulative distribution of trace distances (distance function) (d) at $38 \mathrm{kHz}$. 


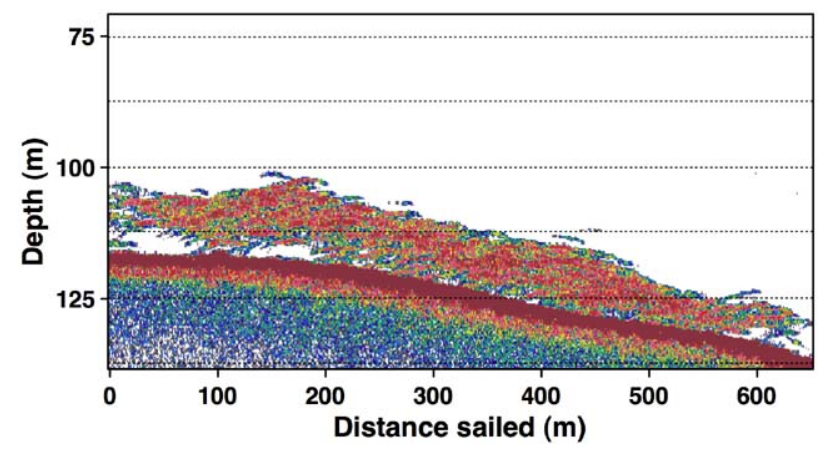

Fig. (5). Echogram of cod over a bottom 110-130 m deep. The distance between the depth lines is $12,5 \mathrm{~m}$.

The trace estimation procedure is to first choose the minimum number of echoes in the traces to be counted, and an average target strength threshold. In this case we count traces with 3 or more echoes, and the threshold is chosen at the value "T" in Fig. (4c) $(-32 \mathrm{~dB})$. Then compute the distance function for all traces with average target strength above the threshold. A straight line is fitted to the central linear part of the distance function as shown in Fig. (4d). Under the assumption that traces are observed from all fish of the target species within the near linear region of the distance function we see that there are 430 traces within $9.5 \mathrm{~m}$ athwarthip width. As these traces are observed over a sailed distance of $779 \mathrm{~m}$, the average fish density is given by $430 /(9.5 \times 779)=0.058 \mathrm{fish} / \mathrm{m}^{2}$.

The same file is now used to demonstrate trace estimation at different frequencies. New threshold values have to be found for each frequency because the scattering properties change with frequency. When comparing estimates at different frequencies, it is important that the threshold values used correspond to each other. This is most easy to achieve when the target species is barely mixed with other species, as is the case with the present file. The lowest frequency, $18 \mathrm{kHz}$, was not tried. The estimation results are given in Table $\mathbf{1}$.

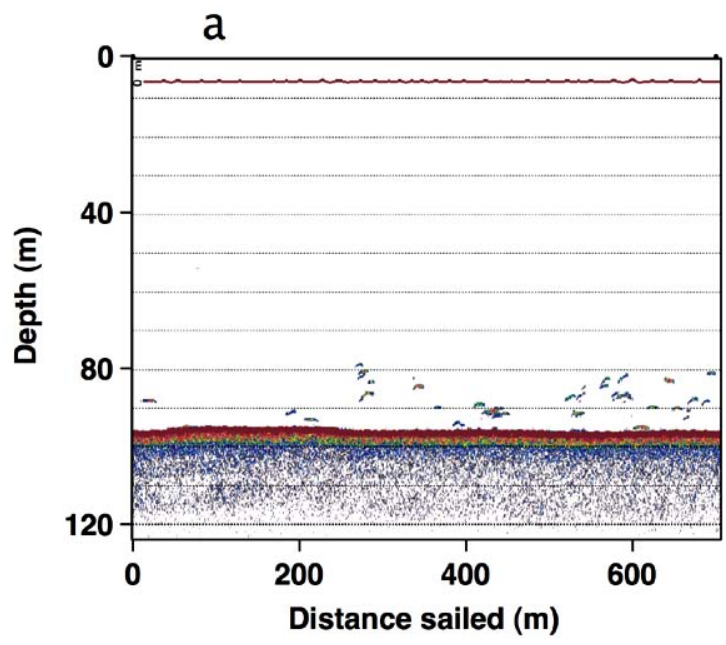

Table 1. Trace Estimates of the Same Fish Density at Four Frequencies. The Likely Reason for a Lower Estimate at $200 \mathrm{kHz}$ is that the Recording is Partly Out of Reach at this Frequency

\begin{tabular}{|c|c|}
\hline Frequency $(\mathbf{k H z})$ & Fish Density Per Square Meters \\
\hline \hline 38 & 0.058 \\
\hline 70 & 0.058 \\
\hline 120 & 0.059 \\
\hline 200 & 0.037 \\
\hline
\end{tabular}

The same file was also run at $38 \mathrm{kHz}$ over the densest concentration shown in Fig. (4a).

This concentration, shown in Fig. (5) is between 105 and $130 \mathrm{~m}$ depth, and more than $600 \mathrm{~m}$ long, and estimation was computed over a distance of $629 \mathrm{~m}$. The echo integration estimate is 0.0614 , and the trace estimate 0.0617 fish per square meters. This is the highest fish density estimated by the trace method so far, but as is explained in the Discussion, the estimates may be too small. However, the volume density in this concentration is of the order of 0.007 fish per cubic meter giving roughly 0.12 fish in the pulse volume on average.

Next, a file with low fish density will be used. This file Fig. (6a), is a near bottom recording where the bottom depths were between 90 and $103 \mathrm{~m}$. The sailing speed varied between 1.4 to $2.8 \mathrm{~m} / \mathrm{s}$ and the distance sailed was $3268 \mathrm{~m}$. The ping repetition rate varied between 1.4 and 2 ping per second. At $38 \mathrm{kHz}$, we obtain:

We see from Fig. (6) that this recording is a mixture of big fish with some smaller individuals that can be filtered away by using suitable threshold values. The integrator estimate is 0.00176 fish per square meter.

This file contains long traces, and the trace estimate is obtained by counting traces with more than 3 echoes, and using the indicated threshold value "T" in Fig. (7a). The line fitted to the distance function in Fig. (7b) gives 0.00180 fish

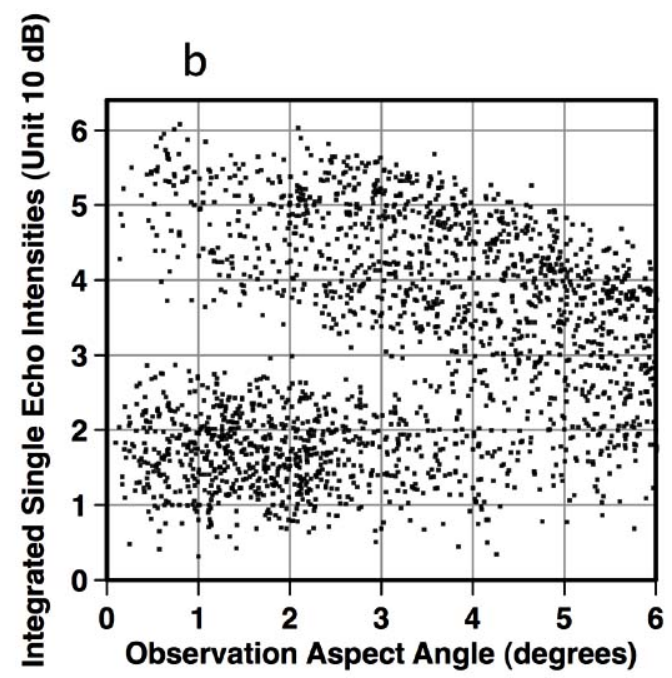

Fig. (6). Echogram with $10 \mathrm{~m}$ between the depth lines (a), and distribution of integrated single fish echo intensities (b) at $38 \mathrm{kHz}$. 

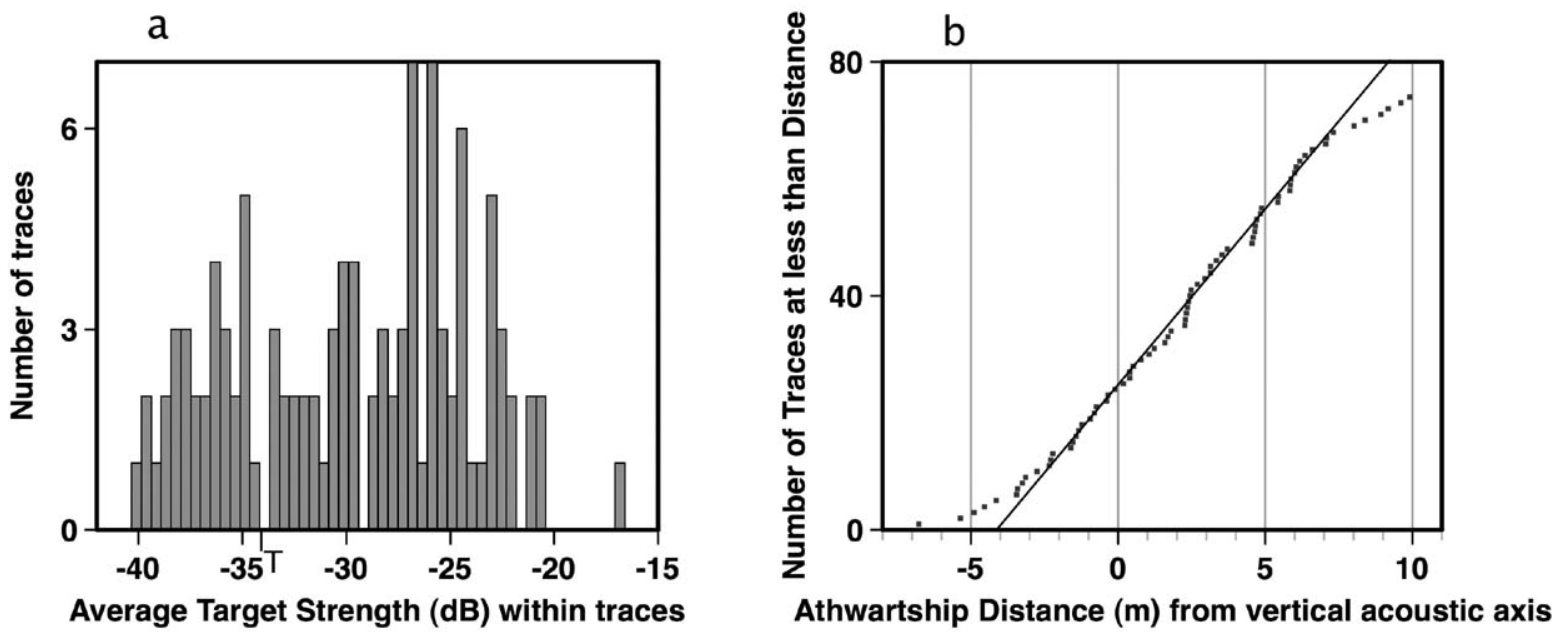

Fig. (7). Distribution of trace Target Strengths with threshold "T" (a), and distance function for traces with more than 3 echoes (b).
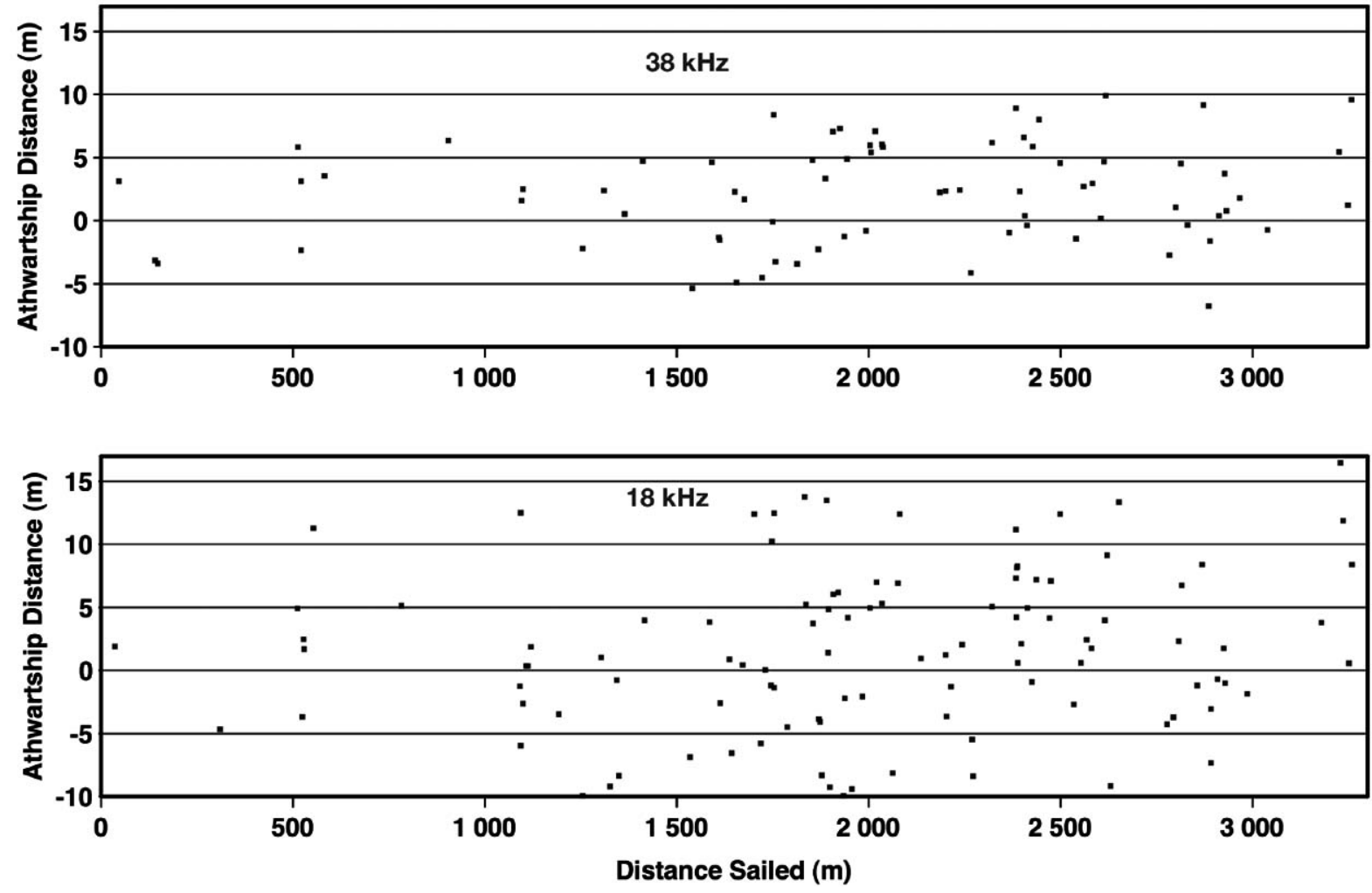

Fig. (8). The local area distribution of fish as observed with $38 \mathrm{kHz}$ (upper) and $18 \mathrm{kHz}$ (lower). The athwartship positions are the average athwartship distance of the traces, and the distance sailed positions are the ship's positions at the times when the center of the traces are detected.

per square meter. The trace threshold is here selected according to the $15 \mathrm{~dB}$ rule. It could alternatively be chosen as -29 $\mathrm{dB}$, but this is too close to the maximum. This file was also tried with $18 \mathrm{kHz}$ with a corresponding trace estimate of 0.00179 fish per square meter.

It is possible to present the positions of all fish that generated a trace. Fig. (8) shows the positions in the area of those fish that were counted at 38 and $18 \mathrm{kHz}$, respectively.
It is seen from Fig. (8) that the $18 \mathrm{kHz}$ transducer has not detected every trace that was detected at $38 \mathrm{kHz}$ although the wider $18 \mathrm{kHz}$ beam is expected to cover the $38 \mathrm{kHz}$ beam completely.

Experience has shown that if the average trace length (number of trace echoes) is low (as occurs during fast sailing speed, slow ping repetition rate, shallow recording, or a combination), the trace method tends to give an underestimate relative to that of echo integration. 


\section{DISCUSSION}

The split beam echo trace method is built on simple principles but has restrictions on the within-beam fish density. To decide whether the method is likely to work well on a recording, assessment of echograms is necessary. With the common split beam systems on research vessels with hullmounted transducers, many dense recordings of fish at some depth are unsuitable for the method. However, if use of acoustic platforms on remotely operated underwater vehicles becomes more common, the range of observation can be chosen. This is also the case for acoustic platforms on towed underwater bodies. For such systems the echo trace method may be used for most recordings.

The echo trace program described here has been developed over some time, and has been upgraded several times. Although Xie (see [11]) has developed an algorithm that sorts the echoes into fish traces, there are several reasons for not using his ideas here. Xie has not implemented a technique to filter away traces from smaller fish and plankton. Instead he uses a fixed echo peak voltage threshold as well as an echo width test to select candidate echoes. The fact that he does not use partly overlapping echoes is one reason why his method does not work under as high fish density as the method presented here. Although the present method is still a prototype, the present level of the program shows that this method is promising. In contrast to the echo integration method, the echo trace method does not require knowledge of fish target strength. Target strength obtained "in situ" is, however, used for trace echoes in this paper, but other signal levels giving values that are proportional to target strength may be used as well.

It is concluded both in [11] and [14] that a good signal to noise ratio is required to analyze echo traces with a split beam system. However, not all echoes in a trace need to be observed in this application. It is sufficient that a trace represents one fish at a reasonably accurate athwartship distance. Also, all fish within some range of athwartship distances have to be observed by several trace echoes.

For the selected files, the trace program has given estimates that are consistent with the echo integration estimates. Many other files have also been tried, but most of these contain a mixture of different species including plankton that made it difficult to choose corresponding threshold values for echo integration and trace estimation. Some had also schools and patches that were too dense to generate trace echoes, and some had too much variation in the sailing speed.

For the chosen files, there were no difficult overlaps in the echo-amplitudes of the big and small individuals.

Use of the distance function (see Fig. 4d) is better than counting the number of traces within some fixed athwartship distance. It was apparent that the echo sounder usually did not "see" echoes equally far on either side of the two athwartship sides of the vertical acoustic axis. The method of fitting a straight line to the "linear" part of the distance function is independent of this phenomenon, which may be caused by different strengths and directions of side wind. The subjective fitting of a straight line to the distance func- tion seems to work well in most cases. An automatic, more objective, line-fitting routine may be developed, but this was not given priority here.

\section{Sources of Bias}

There are several potential sources of bias with the echo trace method. If the test for accepting a new echo to a trace is too strict, some traces may be divided into two or more shorter traces, thus causing overestimation of fish density. If the test is too open, echoes not belonging to the trace may be accepted. However, alien echoes in traces will not cause any bias unless they change the number of counted traces.

There is always a chance that two echoes from different sources, such as a plankton patch or overlapping echoes from other smaller organisms, may be accepted by chance to be the first two echoes in a new trace. Therefore, it is likely that a fraction of false two-echo traces will be generated, as well as a considerably smaller fraction of false three-echo traces. Another way in which additional short traces may be generated is when the first couple of echoes in a trace have similar big errors in echo parameter values while the following echoes have accurate parameter values. Then the trace of the first echoes may be ended as a short trace. It is evident from the distribution of trace lengths that extra short traces may form, in particular when there are many overlapping echoes.

Fig. (9) is from a recording that shows a mixture of big fish with smaller fish and plankton, as seen from the distribution of integrated single echoes. This recording has generated many false short traces. The sailing speed was between 1.4 and $2.4 \mathrm{~m}$ per second.

When trace lengths of more than 10 echoes are observed, there are normally very few real traces spanning over only two or three successive ping numbers. The trace length distributions in Fig. (9) are thus a strong indication of overestimated number of short traces. It can be seen from the difference between the two lower distributions that short traces in number of echoes are distributed over different trace lengths in number of pings. These distributions would have been identical if the echoes in all ping numbers of a trace had been detected.

It can be seen that a few very long traces have been observed. By inspecting the average change in alongship angle per ping, these traces can only have been made by fish that were swimming in the same direction as the acoustic platform during the slowest sailing speed. Trace length patterns with long, thin right-hand tails are often observed when the sailing speed is moderate. Then the fish movements may affect the trace length distribution considerably. The fact that fish are attracted to slow-moving ships and tend to actively stay under the ship has been reported in [22].

If a false echo peak is accepted as the first echo in a new trace, the program will, as long as the next echo has not been found, test whether echoes in the following five pings satisfy the requirement for the next echo. Therefore, false two-echo traces may span up to 6 ping numbers. False traces containing more than two echoes may span even bigger ranges of ping numbers. 

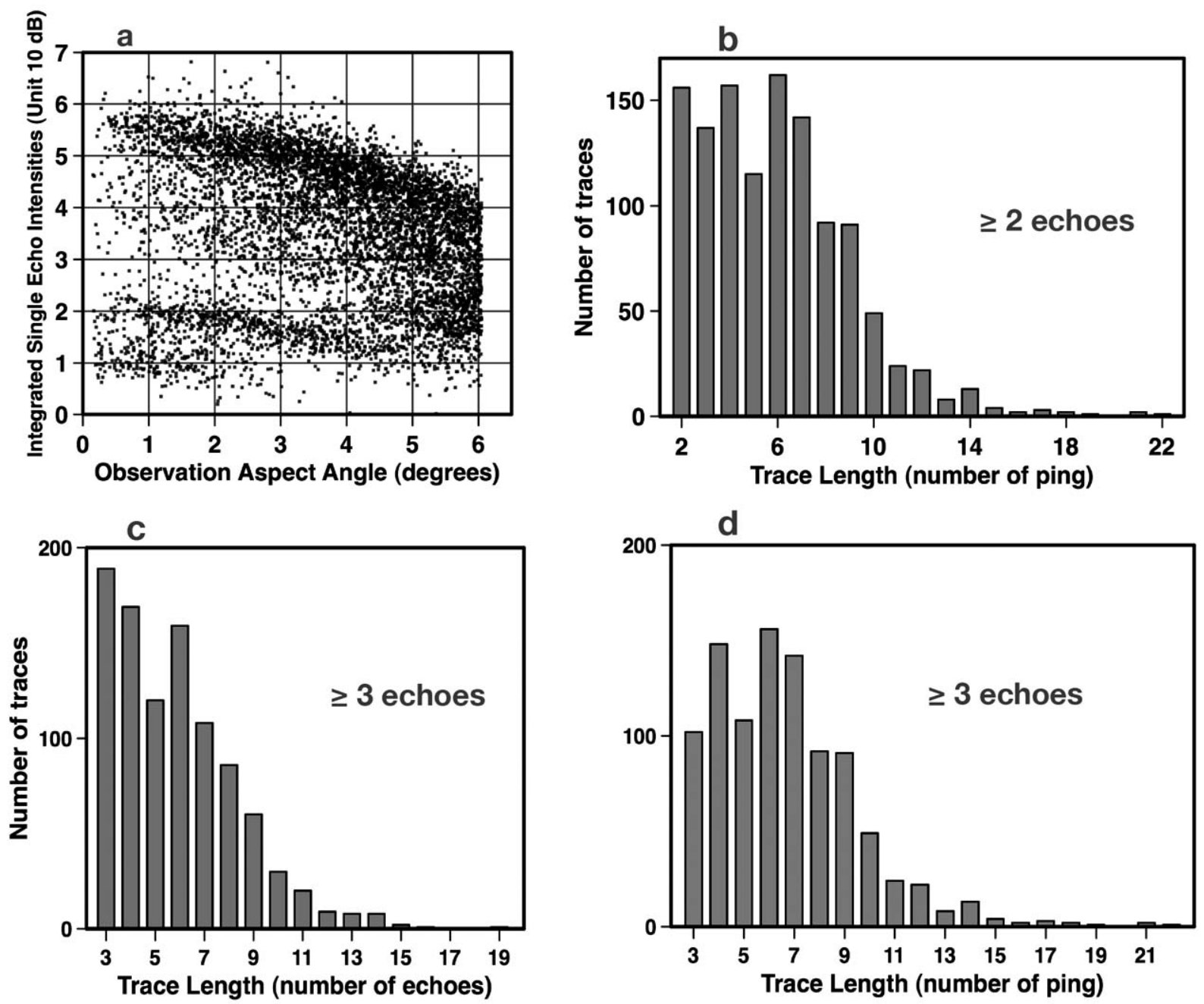

Fig. (9). Integrated single echo intensities (a) and distributions of trace lengths including two-echo traces (b), and with two-echo traces removed (c and d).

The above-described defect of the echo trace program may be reduced. It has already been reduced to some extent relative to earlier versions of the program. Previously, candidate trace echoes were accepted from a wider range of beam angles, but by reducing this, the number of short trace lengths has been reduced. In the present program, candidate echoes is only accepted from targets within 1.5 times the half power opening angle of the transducer.

Also, the value of the parameter $m$ in (3) has been varied with the purpose of reducing the number of short traces, and it was found that a low value of $m$ (weak influence of the theoretical alongship angle slope) gave slightly more short traces, in particular two-echo traces. The value 5 for $m$ has been used in the applications in this paper.

However, to reduce the extra number of short traces more significantly, a more fundamental change in the trace program may be necessary. Some test of echo shape may be helpful, but a difficulty with this is that the extra number of short traces must be reduced without reducing the number of longer traces.

The idea of counting only particular fractions of the shortest traces has been considered, but setting proper values for these fractions is difficult, in particular since the correct fractions are expected to vary with type of recording as well as many observation parameters (sailing speed, ping repetition rate, frequency and so on).

The present program seems to work well when the trace lengths are not too small, by counting traces with 3 or more, or 4 or more, echoes. Since the true number of short traces is a minor part of the total when the average trace length is big, the number of traces except the shortest is a good approximation to the total number of real traces.

Even when the trace estimate agrees with the integrator estimate this does not prove that the trace estimate is correct. In the run over the densest concentration (Fig. 8), the integrator estimate may be too small. This is because more weak 
than strong single echoes are lost when the fish density is high. The conversion factor between integrator value and fish density will then be too big, causing fish density to be underestimated. The degree of this effect here is not clear, but if present, the trace estimate is also too small caused by too high within beam fish density.

\section{Likely Problems with Noise}

The applications in this paper were obtained under a rather good signal to noise ratio. During a worse noise level, e.g. during application on deeper recordings, the first problem will be big errors in the detected off axis angles. When this happens many traces may split into shorter traces, and at even higher noise levels many traces are not observed at all. Although a few of the available files of raw data contained recordings at depths down to $400-500 \mathrm{~m}$, this was not enough to study the behavior of the echo trace program at different critical noise levels.

If the trace program is to be used with higher noise level, it may work better on low fish density if the maximal distances between the echo parameters of a new echo and the expected parameters of the next echo are bigger. When the errors in the off axis angles are big, valid traces may then be formed without splitting traces. This is an argument for having these distance values as input parameters to the program. It would be an easy update.

\section{Further Development}

Further development of the trace program will be necessary for different reasons. Reduction of known defects is obvious, but adaptation to future and hopefully better versions of the split beam echo sounder system is also necessary. The present program reads the echo signals sequentially without going back to any previous ping.
An alternative way to build traces would be to start with strong echoes more centrally in the beam and search for accompanying trace echoes in pings both backward and forward in time. This has not been tried here, but it may be a way to build traces without many extra short traces.

\section{CONCLUSIONS}

Estimates of fish density are obtained by software that counts echo traces. Most of these are consistent with corresponding estimates obtained by echo integration.

The trace method is often encumbered with overestimated numbers of short traces, in particular two echo traces. To get rid of these false traces, counting of traces with 3 or more, or 4 or more, echoes is recommended.

When the trace lengths are small, usually when all traces contain less than 10 echoes, the trace method tends to underestimate fish density.

Advantages with the trace method are that it represents a method by which echo integration estimates may be compared, that it can be used on species with unknown Target Strengths and that it can be used without calibrated echo sounders, although with approximately known beam functions.

At present, it is not found any situation where the trace method is definitely better than the echo integration method. However, such situations may be found in the future.

\section{ACKNOWLEDGEMENTS}

I want to thank Egil Ona, Institute of Marine Research (IMR), Bergen, Norway, for providing the files of acoustic raw data used to develop this method.

\section{APPENDIX A}

\section{A Flow Chart and Some Technical Information About the Program}

The program is structured in terms of four main subroutines. "ReadPing" reads the next ping, computes the echo signal data with both 40 and $20 \log$ TVG and computes the associated phase angle data.

"ScanPing" scans the echo signal for echo peaks and test whether the associated echoes belong to some unfinished traces, or are the first echo of a new trace.

"PutTrace" updates each unfinished trace with new echoes from the last ping, and create new traces with a first echo if necessary.

"EndTrace" test each existing trace structure for being finished. For traces that satisfy the condition for being finished the routine computes average trace parameters such as the echo depth, the echo amplitude, the athwartship angle and the athwartship distance between the vertical acoustic axis and the target.

A simple Flow Chart describing the trace program is shown in Fig. (10). 


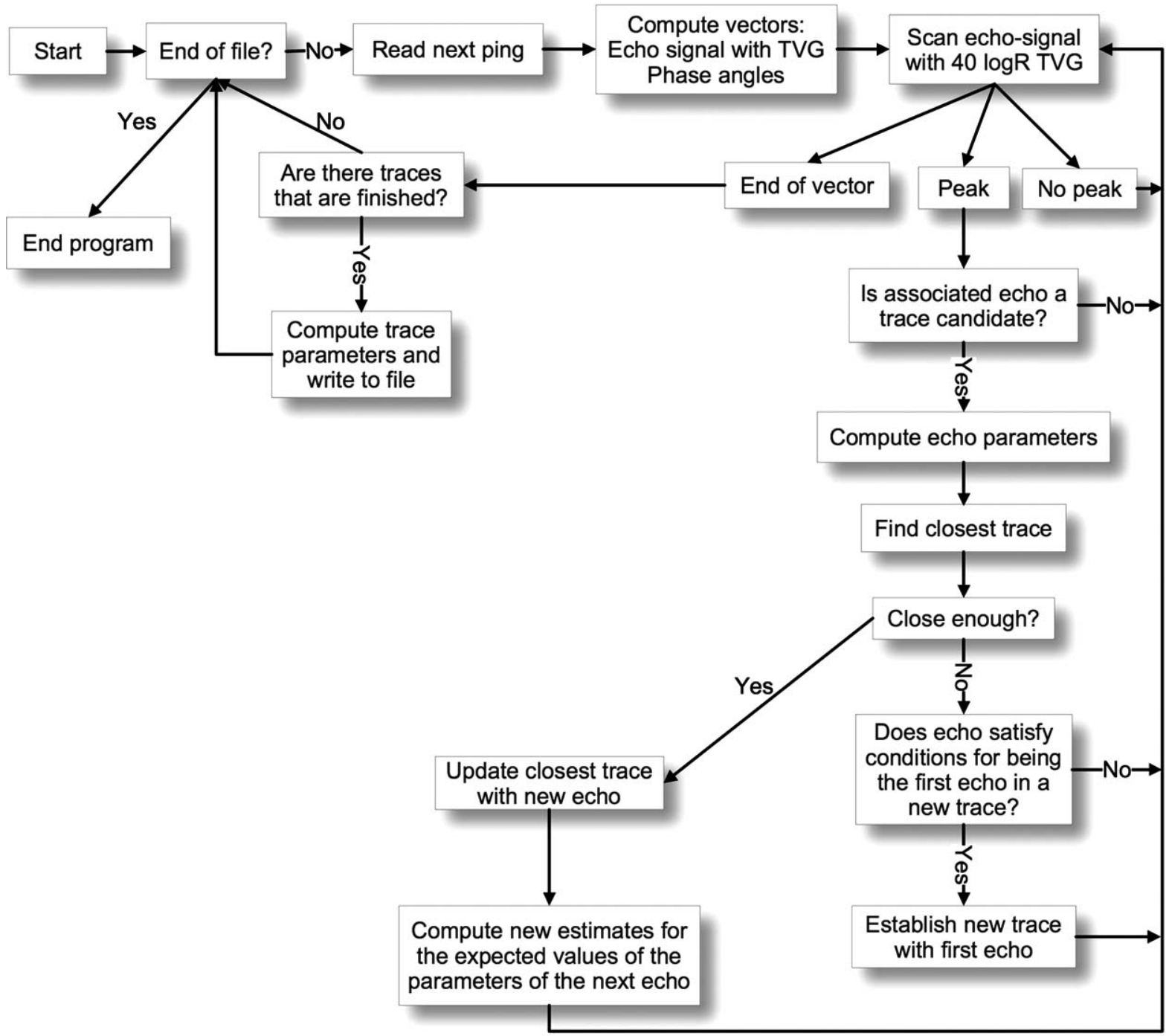

Fig. (10). Flow Chart of the trace program. Echo integration is not shown.

During program start several datagram containing necessary parameters in the file of raw data are read as well as the user defined parameter values. The data structure of the raw data files is needed for this, and is given in the help menu of the SIMRAD EK60 and ER60 echo sounder software.

During program development the number of user specified parameters has increased. At present the parameter file contain values for the following 14 parameters.

$n_{\text {min }}$ (minimum number of echoes in counted traces)

file number (different files of raw data are associated with a set of file numbers)

frequency number $(0=18 \mathrm{kHz}, 1=38 \mathrm{kHz}, 2=70 \mathrm{kHz}, 3=120 \mathrm{kHz}$ and $4=200 \mathrm{kHz})$

]lower trace threshold $(\mathrm{dB})$

upper trace threshold $(\mathrm{dB})$

upper depth (m)

lower depth (m)

maximum distance above the bottom (m)

lower ping number 
upper ping number

single integrated echo threshold (for echo integration conversion factor) (special unit)

echo-signal threshold for integration (special unit)

a parameter related to how close to the bottom echo-signal integration is carried out $m$ in (3)

Most of these parameters limit the region where echo integration and trace formation are carried out. This is important for being able to "see" a clear threshold value (see Fig. 3).

The trace program uses two threshold values where the lowest has to be exceeded by a beam-compensated echo peak to be used as a candidate for echo traces. The other threshold has to be exceeded by the average beam compensated echo intensity over the echoes in a finished echo trace for this to be counted.

The beam-compensations of echoes are based on a fitted Bessel function model of the beam shape as described in [21]. The inverse of the average beam damping in each of fourteen equal angle intervals out to around $-20 \mathrm{~dB}$ transmit/receive damping is used as beam-compensation factors. This range of angles is bigger than the range used for possible candidate trace echoes, thus permitting the use of bigger ranges for candidate echoes.

Scanning the echo signal is done by first reading three consecutive sample values. This is then continued by reading the next sample value and discarding the first.

During execution, echo traces are stored in structures containing the values of each echo parameter of each echo in the trace. In addition, several vectors outside the trace structure are indexed by trace number and contain special variables associated with the traces. Examples are the number of echoes presently in the trace, the weighted average depth of the echoes in the trace, the weighted average echo intensity of the echoes in the trace and so on, as well as estimates of the expected values of the parameters of the next echo for the trace. These last variables are essential for deciding which echo is to be taken as the next in the trace.

The condition for ending unfinished traces depends on how many pings that has elapsed without finding any new echo belonging to the trace. In the program so far, if more than four pings have elapsed after the last echo, this trace is taken to be finished. An effect of this is that no trace will have gaps of more than four ping numbers in the ping numbers of its echoes.

The "End of vector" and "End of file" events in the Flow Chart usually happen before end of file or end of vector is reached. This is because user determined parameters may limits the number of pings and depth interval searched by the program.

End program does final computations on the integrator estimate and write out necessary parameter values. The trace estimate is obtained by making the distance function from one data file made by the program.

\section{Noise}

The signal to noise ratio for the used split beam system is very good. To illustrate the noise level, the signal intensities of the reflected signal from around $100 \mathrm{~m}$ depth from one file of raw data that contained cod echoes at this depth were plotted and are shown in Fig. (11).

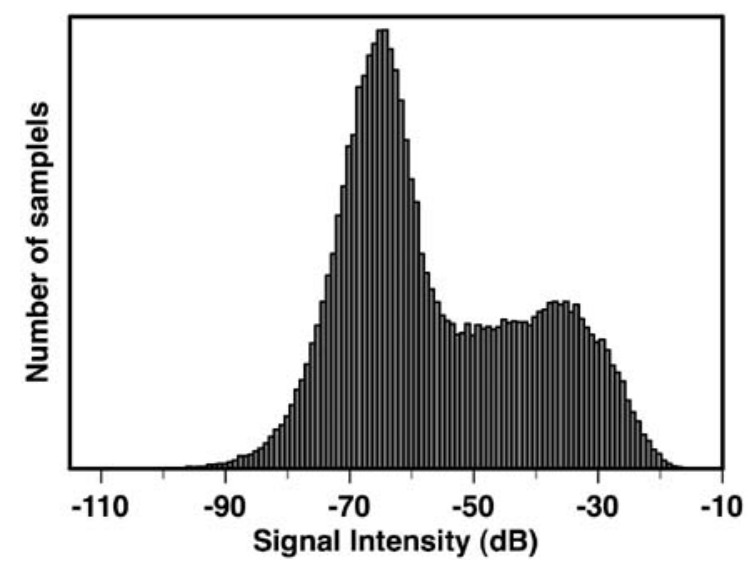

Fig. (11). Signal and noise histograms; All signal sample values with $40 \log \mathrm{R}$ TVG from between 95 and $105 \mathrm{~m}$ depth at $70 \mathrm{kHz}$ were used. The leftmost peak represents noise and weak reverberation; the region between the right peak and tail represents cod including overlapping echoes while the intermediate region represents reverberation from smaller organisms as well as weak echoes far out in the beam. Corresponding plots for the other frequencies are similar, but shifted to higher values for lower frequencies and vice versa. 


\section{APPENDIX B}

Example of traces with Echo Parameters Generated by the Trace Program

A section of a file containing the echo parameters within each trace is shown in Table 2.

Table 2. The Parameters of Two Echo Traces. The Echo Amplitudes are Here Expressed in Some Absolute Unit Giving High Values.

The Unit $\propto v^{2}$ Means Proportional to Peak Voltage Squared. Beam Compensation is Without Dimension.

Trace 1

Mean athwartship angle: -3.4576

Distance from acoustic axis: -4.1864

Mean echo amplitude: 120808

Number of echoes: 18

\begin{tabular}{|c|c|c|c|c|c|c|c|c|c|}
\hline $\begin{array}{c}\text { Ping } \\
\text { Number }\end{array}$ & $\begin{array}{c}\text { Echo } \\
\text { Depth } \\
\text { m }\end{array}$ & $\begin{array}{c}\text { Bottom } \\
\text { Depth } \\
\text { m }\end{array}$ & $\begin{array}{l}\text { Echo } \\
\text { Ampl. } \\
\propto v^{2}\end{array}$ & $\begin{array}{l}\text { Beam } \\
\text { comp. }\end{array}$ & $\begin{array}{c}\text { Alongship } \\
\text { Angle } \\
\text { Degrees }\end{array}$ & $\begin{array}{c}\text { Instability } \\
\text { Index } \\
\text { Degrees }\end{array}$ & $\begin{array}{c}\text { Athwartship } \\
\text { Angle } \\
\text { Degrees }\end{array}$ & $\begin{array}{c}\text { Instability } \\
\text { Index } \\
\text { Degrees }\end{array}$ & $\begin{array}{c}\text { Speed } \\
\mathbf{m} / \mathbf{s}\end{array}$ \\
\hline 3060 & 69.69 & 77.33 & 20812 & 81.82 & 6.0 & 0.45 & -3.2 & 1.18 & 3.35 \\
\hline 3061 & 69.50 & 77.52 & 41706 & 81.82 & 5.7 & 0.17 & -3.7 & 0.13 & 3.34 \\
\hline 3062 & 69.59 & 77.52 & 110840 & 81.82 & 5.4 & 0.04 & -3.0 & 0.00 & 3.33 \\
\hline 3063 & 69.41 & 77.52 & 136057 & 81.82 & 5.1 & 0.06 & -3.3 & 0.04 & 3.33 \\
\hline 3064 & 69.46 & 77.52 & 275079 & 81.82 & 4.6 & 0.04 & -3.4 & 0.04 & 3.33 \\
\hline 3065 & 69.53 & 77.52 & 180754 & 19.74 & 3.9 & 0.06 & -3.4 & 0.04 & 3.33 \\
\hline 3066 & 69.39 & 77.71 & 87803 & 11.26 & 3.4 & 0.09 & -3.2 & 0.00 & 3.33 \\
\hline 3067 & 69.41 & 77.90 & 74637 & 11.26 & 3.1 & 0.06 & -3.3 & 0.04 & 3.33 \\
\hline 3068 & 69.44 & 77.71 & 109729 & 6.94 & 2.4 & 0.04 & -3.4 & 0.04 & 3.33 \\
\hline 3069 & 69.28 & 77.71 & 79481 & 4.58 & 2.0 & 0.06 & -3.3 & 0.04 & 3.33 \\
\hline 3070 & 69.28 & 77.90 & 146164 & 4.58 & 1.5 & 0.04 & -3.5 & 0.00 & 3.33 \\
\hline 3071 & 69.29 & 77.90 & 131873 & 4.58 & 0.9 & 0.00 & -3.5 & 0.00 & 3.33 \\
\hline 3072 & 69.30 & 78.09 & 91814 & 3.20 & 0.6 & 0.04 & -3.4 & 0.00 & 3.33 \\
\hline 3073 & 69.12 & 78.09 & 83215 & 3.20 & 0.1 & 0.06 & -3.4 & 0.04 & 3.33 \\
\hline 3074 & 69.09 & 77.90 & 148027 & 4.58 & -0.5 & 0.04 & -3.7 & 0.00 & 3.33 \\
\hline 3075 & 69.04 & 78.09 & 203087 & 6.94 & -1.2 & 0.00 & -4.2 & 0.00 & 3.33 \\
\hline 3076 & 69.08 & 78.09 & 82257 & 4.58 & -1.3 & 0.04 & -3.7 & 0.00 & 3.33 \\
\hline 3077 & 68.91 & 78.09 & 51585 & 4.58 & -1.5 & 0.09 & -3.4 & 0.21 & 3.33 \\
\hline
\end{tabular}


Trace2

Mean athwartship angle: 1.1037

Distance from acoustic axis: 1.2468

Mean echo amplitude: 111358

Number of echoes: 8

\begin{tabular}{|c|c|c|c|c|c|c|c|c|c|}
\hline $\begin{array}{c}\text { Ping } \\
\text { Number }\end{array}$ & $\begin{array}{c}\text { Echo } \\
\text { Depth } \\
\text { m }\end{array}$ & $\begin{array}{c}\text { Bottom } \\
\text { Depth } \\
\text { m }\end{array}$ & $\begin{array}{l}\text { Echo } \\
\text { Ampl. } \\
\propto v^{2}\end{array}$ & $\begin{array}{l}\text { Beam } \\
\text { Comp. }\end{array}$ & $\begin{array}{l}\text { Alongship } \\
\text { Angle } \\
\text { Degrees }\end{array}$ & $\begin{array}{c}\text { Instability } \\
\text { Index } \\
\text { Degrees }\end{array}$ & $\begin{array}{c}\text { Athwartship } \\
\text { Angle } \\
\text { Degrees }\end{array}$ & $\begin{array}{c}\text { Instability } \\
\text { Index } \\
\text { Degrees }\end{array}$ & $\begin{array}{c}\text { Speed } \\
\mathbf{m} / \mathbf{s}\end{array}$ \\
\hline 3068 & 64.91 & 77.71 & 83724 & 11.26 & 4.0 & 0.06 & 1.7 & 0.34 & 3.33 \\
\hline 3072 & 64.84 & 78.09 & 63604 & 2.37 & 2.3 & 0.39 & 1.6 & 1.71 & 3.33 \\
\hline 3073 & 64.71 & 78.09 & 110864 & 1.27 & 0.9 & 0.75 & 1.2 & 0.96 & 3.33 \\
\hline 3074 & 64.71 & 77.90 & 76069 & 1.13 & 0.0 & 0.49 & 1.2 & 0.45 & 3.33 \\
\hline 3078 & 64.50 & 78.09 & 109717 & 1.50 & -1.6 & 0.11 & 1.1 & 0.04 & 3.33 \\
\hline
\end{tabular}

Table 2 shows two traces. The first with 18 echoes has rather stable angle observations while the second has more unstable angles. Unstable angles of an echo occur when there is other echoes close by. Fig. (12) shows that this is the case with the last trace in Table 2.

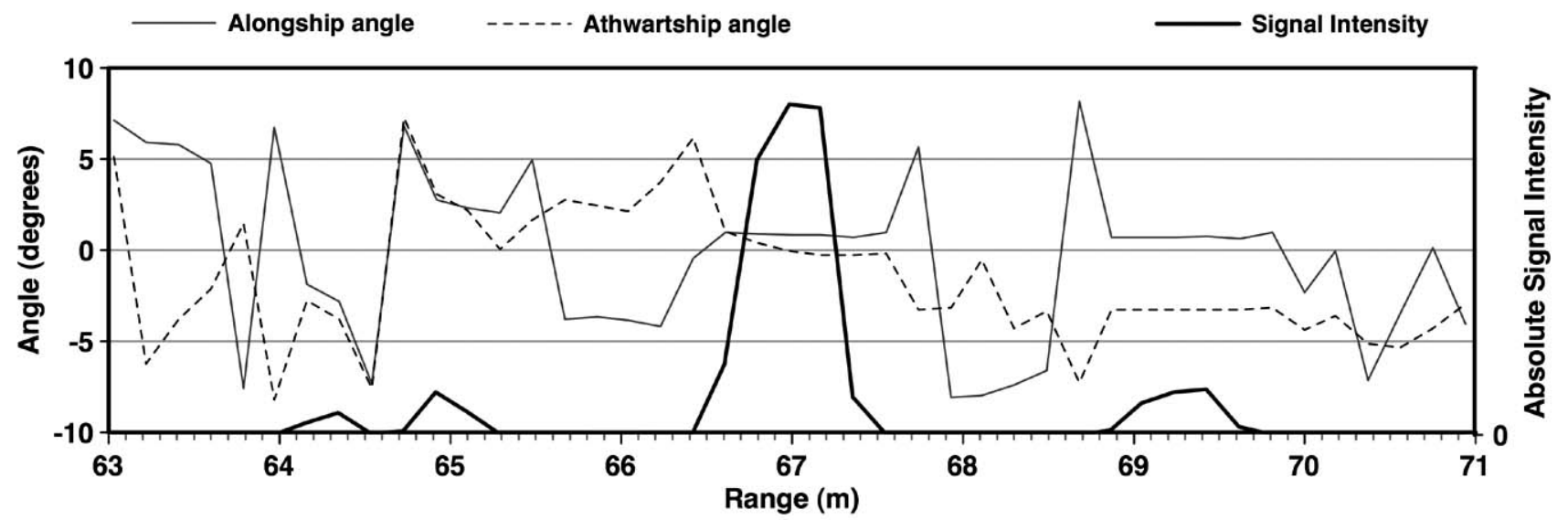

Fig. (12). Graphical presentation of the echo-signal intensity (absolute values) and angle signals in a part of ping number 3072 that is contained in both traces in Table 1. The graph is made from the signal sample values. The reason for no labels on the Signal Intensity axis is the same as in Fig. (1).

The echo at a range close to $65 \mathrm{~m}$ belongs to the second trace in Table 2 , and it can be seen that the angle signals are rather variable over this echo. The likely cause is influence from the echo just above as well as from a weaker echo between these two. The echo slightly below $69 \mathrm{~m}$ in Fig. (12) has very stable angles and belongs to the first trace in Table 2.

\section{REFERENCES}

[1] O. Sund, "Echo-sounding in fishery research", Nature, vol. 135, pp. 473-475, 1935.

[2] R. B. Mitson, and R. J. Wood, "An automatic method of counting fish echoes", Fisheries Laboratory, Lowestoft, 1961.

[3] R.E. Craig, and S.T. Forbes, "Design of a sonar for fish counting", Fisk Dir. Skr. Ser. HavUnders, vol. 15, pp. 210-219, 1969.
[4] R. Kieser, and T. J. Mulligan, "Analysis of echo counting data: a model”, Can. J. Fish. Aquat. Sci., vol. 41, pp. 451-458, 1984.

[5] K. G. Foote, "Importance of the swimbladder in acoustic scattering by fish: a comparison of godoid and mackerel target strengths " $J$. Acoust. Soc. Am., vol. 67, pp. 2084-2089, 1980.

[6] D. N. MacLennan, "Acoustical measurement of fish abundance", $J$. Acoust. Soc. Am., vol. 87(1), pp. 1 -15, 1990. 
[7] R. J. Korneliussen, "The Bergen echo integrator post processing system, with focus on recent improvements", Fish. Res., vol. 68, pp. 159-169, 2004.

[8] M.V. Trevorrow, D.M. Farmer, and B. Pedersen, "Shallow-water salmon and herring detection using $100-\mathrm{kHz}$ sidescan sonars", $J$. Acoust. Soc. Am., vol. 103, pp. 2939-2939, 1998.

[9] T. Mulligan, and R. Kieser, "A split-beam echo counting model for riverine use”, ICES J. Mar. Sci., vol. 53, pp. 403-406, 1996.

[10] T. J. Mulligan, and D. G. Chen, "A split-beam echo counting model: development of statistical procedures", ICES J. Mar. Sci., vol. 55, pp. 905-917, 1998.

[11] Y. Xie, "A range-dependent echo-association algorithm and its application in split-beam sonar tracking of migratory salmon in the Fraser River watershed", IEEE J. Oceanic Eng., vol. 25, no. 3, pp. 387-398, 2000.

[12] K. Sawada, Y. Miyanohana, H. Takahashi, and Y. Takao, "Observation of fish length and behaviour of an individual fish using echo trace analysis", J. Acoust. Soc. Am., vol.109, p. 2939, 2001.

[13] K. Abe, Y. Takao, K. Sadayasu, K. Sawada, and K. Amakasu, "Target strength and behavior of swimming juvenile southern bluefin tuna in a cage estimated by echo-trace analysis", J. Acoust. Soc. Am., vol. 120, p. 3106, 2006.
[14] M. Furusawa, and K. Amakasu, "Robust analysis of single echo traces obtained by split beam sounder", J. Acoust. Soc. Am., vol. 120, p. 3060, 2006.

[15] N. O. Handegard, R. Patel, and V. Hjellvik, "Tracking individual fish from a moving platform using a split-beam transducer", $J$ Acoust. Soc. Am., vol. 118, pp. 2210-2223, 2005.

[16] N. O. Handegard, "Observing individual fish behavior in fish aggregations: tracking in dense fish aggregations using a split-beam echosounder", J. Acoust. Soc. Am., vol. 122, pp. 177-187, 2007.

[17] P. Reynisson, "Split-beam method", in Methodology for TargetStrength Measurements (with Special Reference to In Situ Techniques for Fish and Mikro-nekton) (ch. 6), Ed. E. Ona. ICES Cooperative Research Report. 235 pp., 1999.

[18] O. Nakken, and K. Olsen, "Target strength measurements of fish", Rapp. P. - v. Cons. Int. Explor. Mer., vol. 170, pp. 52-69, 1977.

[19] S. S. Blackman and R. Popoli, Design and analysis of modern tracking systems. Artech House, Massachusetts, 1999.

[20] M. Aksland, "Basic echo-trace-length statistics", ICES C.M. 1985/B:32 Sess. Y., 7 pp., 1985.

[21] M. Aksland, "Applying an alternative method of echo-integration", ICES J. Mar. Sci., vol. 63, pp. 1438-1452, 2006.

[22] A. Røstad, S. Kaartvedt, T. A. Klevjer, and W. Melle, "Fish are attracted to vessels", ICES J. Mar. Sci., vol. 63, pp. 1431-1437, 2006.

(C) Magnar Aksland; Licensee Bentham Open.

This is an open access article licensed under the terms of the Creative Commons Attribution Non-Commercial License (http://creativecommons.org/licenses/by-nc/3.0/) which permits unrestricted, non-commercial use, distribution and reproduction in any medium, provided the work is properly cited. 